\title{
A GENERALIZATION OF MESHULAM'S THEOREM ON SUBSETS OF FINITE ABELIAN GROUPS WITH NO 3-TERM ARITHMETIC PROGRESSION (II)
}

\author{
YU-RU LIU, CRAIG V. SPENCER, AND XIAOMEI ZHAO
}

\begin{abstract}
Let $G \simeq \mathbb{Z} / k_{1} \mathbb{Z} \oplus \cdots \oplus \mathbb{Z} / k_{N} \mathbb{Z}$ be a finite abelian group with $k_{i} \mid k_{i-1}(2 \leq i \leq N)$. For a matrix $Y=\left(a_{i, j}\right) \in \mathbb{Z}^{R \times S}$ satisfying $a_{i, 1}+\cdots+a_{i, S}=0(1 \leq i \leq R)$, let $D_{Y}(G)$ denote the maximal cardinality of a set $A \subseteq G$ for which the equations $a_{i, 1} x_{1}+\cdots+a_{i, S} x_{S}=0$ $(1 \leq i \leq R)$ are never satisfied simultaneously by distinct elements $x_{1}, \ldots, x_{S} \in A$. Under certain assumptions on $Y$ and $G$, we prove an upper bound of the form $D_{Y}(G) \leq|G|(C / N)^{\gamma}$ for positive constants $C$ and $\gamma$.
\end{abstract}

\section{INTRODUCTION}

Let $G$ be a finite abelian group, and let $D_{3}(G)$ denote the maximal cardinality of a subset $A \subseteq G$ which does not contain a 3 -term arithmetic progression. Let $k \in \mathbb{N}=\{1,2, \ldots\}$ with $\operatorname{gcd}(2, k)=1$. In his fundamental paper [9], Roth proved that $D_{3}(\mathbb{Z} / k \mathbb{Z})=O(k / \log \log k)$. His result was later improved by Heath-Brown [6] and Szemerédi [11] to $D_{3}(\mathbb{Z} / k \mathbb{Z})=O\left(k /(\log k)^{\alpha}\right)$ for some small positive constant $\alpha>0$. Recently, Bourgain [2] proved that $D_{3}(\mathbb{Z} / k \mathbb{Z})=$ $O\left(k(\log \log k)^{2} /(\log k)^{2 / 3}\right)$, which provides the best bound currently known. For a general finite abelian group $G$ of odd order, Brown and Buhler [1] and Frankl, Graham, and Rödl [3] showed that $D_{3}(G)=o(|G|)$. In [8], Meshulam considered the case where $G$ has many constituents, and he proved that $D_{3}(G) \leq 2|G| / c(G)$, where $c(G)$ denotes the number of constituents of $G$. By combining Meshulam's result with Bourgain's bound, one can follow the proof of [8, Corollary 1.3] to obtain that $D_{3}(G)=O\left(|G| /(\log |G|)^{\beta}\right)$, where $\beta$ is any positive constant with $\beta<2 / 5$. By adapting Bourgain's argument in [2] to a general finite abelian group $G$ of odd order, one should in fact be able to prove that $D_{3}(G)=O\left(|G| /(\log |G|)^{\beta}\right)$, where $\beta$ is any positive constant with $\beta<2 / 3$. In [7], the first two authors of this paper generalized Meshulam's result to give an upper bound for subsets of finite abelian groups which avoid non-trivial solutions to a linear equation of the form $r_{1} x_{1}+r_{2} x_{2}+\cdots+r_{s} x_{s}=0$. In this paper, we follow the approaches of [7] and [10] to further generalize Meshulam's result by investigating solutions of a system of equations.

Date: October 15, 2010.

2000 Mathematics Subject Classification. 11T24, 20 D60.

Key words and phrases. Roth's theorem, finite abelian groups, character sums.

The research of the first author is supported in part by an NSERC discovery grant.

The research of the second author is supported in part by NSF Grant DMS-0635607 and NSA Young Investigators Grant H98230-10-1-0155. 
Given a finite abelian group $G$, we can write

$$
G \simeq \mathbb{Z} / k_{1} \mathbb{Z} \oplus \cdots \oplus \mathbb{Z} / k_{M} \mathbb{Z}
$$

where $\mathbb{Z} / k_{i} \mathbb{Z}$ is a non-trivial cyclic group of order $k_{i}(1 \leq i \leq M)$ and $k_{i} \mid k_{i-1}(2 \leq i \leq M)$. We denote by $c(G)=M$ the number of constituents of $G$ and by $a(G)=k_{1}$ the annihilator of $G$. For $R, S \in \mathbb{N}$ with $S \geq 2 R+1$, let $Y=\left(a_{i, j}\right)$ be an $R \times S$ matrix whose elements are integers. Let $L \in \mathbb{N}$ with $L \geq R$. We say that the group $G$ is $L$-coprime to $Y$ if there exist $L$ columns of $Y$ such that:

- any $R$ of these $L$ columns form a matrix of determinant coprime to $a(G)$,

- after removing any $L-R+1$ of these $L$ columns from $Y$, we can find two disjoint sets of $R$ columns which form matrices of determinant coprime to $a(G)$.

In this case, we denote by $\mathcal{I}_{Y}(G ; L)$ the set of indices of these $L$ columns. The $L$-coprimality condition on $Y$ is essential for the arguments of this paper. In order to study systems of higher complexity, one could use higher-order Fourier analysis (see, for example, $[4,5]$ ).

Let $Y=\left(a_{i, j}\right) \in \mathbb{Z}^{R \times S}$ satisfy $a_{i, 1}+\cdots+a_{i, S}=0(1 \leq i \leq R)$. Consider the system of equations

$$
a_{i, 1} x_{1}+\cdots+a_{i, S} x_{S}=0 \quad(1 \leq i \leq R) .
$$

Let $D_{Y}(G)$ denote the maximal cardinality of a set $A \subseteq G$ for which the equations in (1) are never satisfied simultaneously by distinct elements $x_{1}, \ldots, x_{S} \in A$, and let $|G|$ denote the cardinality of $G$. For $L, N \in \mathbb{N}$ with $L \geq R$, we denote by $d_{Y}(N ; L)$ the supremum of $D_{Y}(G)|G|^{-1}$ as $G$ ranges over all finite abelian groups with $c(G) \geq N$ that are $L$-coprime to $Y$. In this paper, we prove the following theorem.

Theorem 1. For $R, S \in \mathbb{N}$ with $S \geq 2 R+1$, let $Y=\left(a_{i, j}\right) \in \mathbb{Z}^{R \times S}$ satisfy $a_{i, 1}+\cdots+a_{i, S}=0$ $(1 \leq i \leq R)$. For $L \in \mathbb{N}$ with $L \geq R$, there exists an effectively computable constant $C=$ $C(Y ; L)>1$ such that for $N \in \mathbb{N}$, we have

$$
d_{Y}(N ; L) \leq\left(\frac{C}{N}\right)^{\frac{L-R+1}{R}}
$$

We note that in the special case when $L=R$, the above conditions on $G$ and $Y$ are analogous to Conditions 1 and 2 in [10]. Hence, Theorem 1 is more general than the finite abelian group analogue of Roth's result in [10]. Also, in the special case when $R=1$ and $L=S-2$, we can derive [7, Theorem 1] from Theorem 1 (see Remark 1). In particular, if $Y=(1,-2,1$ ) (thus $L=R=1$ and $G$ is of odd order), by [7, Remark 6], the constant $C$ in Theorem 1 can be taken to be 2 . Thus, Theorem 1 implies Meshulam's result on subsets of finite abelian groups with no 3-term arithmetic progression [8, Theorem 1.2].

We conclude this section by recalling some properties of character sums of finite abelian groups. Let $\hat{G}$ denote the character group of $G$. For $g \in G$, we have

$$
|G|^{-1} \sum_{\chi \in \hat{G}} \chi(g)= \begin{cases}1, & \text { if } g=0, \\ 0, & \text { otherwise. }\end{cases}
$$


For $R \in \mathbb{N}$, the character group of $G^{R}$ is equivalent to the product of $R$ copies of $\hat{G}$, and we denote it by $\hat{G}^{R}$. Thus, for $\chi=\left(\chi_{1}, \ldots, \chi_{R}\right) \in \hat{G}^{R}$ and $\left(g_{1}, \ldots, g_{R}\right) \in G^{R}$, we have

$$
\begin{aligned}
|G|^{-R} \sum_{\chi \in \hat{G}^{R}} \chi_{1}\left(g_{1}\right) \cdots \chi_{R}\left(g_{R}\right) & =\prod_{i=1}^{R}\left(|G|^{-1} \sum_{\chi_{i} \in \hat{G}} \chi_{i}\left(g_{i}\right)\right) \\
& = \begin{cases}1, & \text { if } g_{j}=0(1 \leq j \leq R), \\
0, & \text { otherwise. }\end{cases}
\end{aligned}
$$

In what follows, we will write $\mathbf{1}$ for the trivial character $(1, \ldots, 1) \in \hat{G}^{R}$ and $\Gamma(G)$ for $\hat{G}^{R} \backslash\{\mathbf{1}\}$.

Acknowledgements The authors would like to thank the referees for providing valuable suggestions and comments.

\section{Proof of Theorem 1}

For $R, S \in \mathbb{N}$ with $S \geq 2 R+1$, let $Y=\left(a_{i, j}\right) \in \mathbb{Z}^{R \times S}$ satisfy $a_{i, 1}+\cdots+a_{i, S}=0(1 \leq i \leq R)$. For $L, N \in \mathbb{N}$ with $L \geq R$, let $G$ be a finite abelian group with $c(G) \geq N$ that is $L$-coprime to $Y$. Let $D_{Y}(G)$ and $d_{Y}(N ; L)$ be defined as in Section 1 . For convenience, in what follows, we will write $D(G)$ in place of $D_{Y}(G)$ and $d(N)$ in place of $d_{Y}(N ; L)$. For a set $A \subseteq G$, let $T(A)=T_{Y}(A)$ denote the number of solutions of (1) with $x_{i} \in A(1 \leq i \leq S)$. For $1 \leq j \leq S$ and $\chi=\left(\chi_{1}, \ldots, \chi_{R}\right) \in \hat{G}^{R}$, define

$$
F_{j}(\chi)=F_{j}(\chi ; A)=\sum_{x \in A} \chi_{1}\left(a_{1, j} x\right) \cdots \chi_{R}\left(a_{R, j} x\right)=\sum_{x \in A} \chi_{1}^{a_{1, j}} \cdots \chi_{R}^{a_{R, j}}(x) .
$$

Then by (2), we have

$$
\begin{aligned}
T(A) & =|G|^{-R} \sum_{\chi \in \hat{G}^{R}} F_{1} \cdots F_{S}(\chi) \\
& =|G|^{-R} F_{1} \cdots F_{S}(\mathbf{1})+|G|^{-R} \sum_{\chi \in \Gamma(G)} F_{1} \cdots F_{S}(\chi) .
\end{aligned}
$$

Before proving Theorem 1, we will need to obtain bounds on $T(A)$ and the contribution of the non-trivial characters.

Lemma 2. Let $G$ be a finite abelian group. For $R \in \mathbb{N}$, let $Z \in \mathbb{Z}^{R \times R}$ satisfy $\operatorname{gcd}(\operatorname{det} Z, a(G))=$ 1 , where $\operatorname{det} Z$ denotes the determinant of $Z$. For $\mathbf{x} \in G^{R}$, we have $Z \mathbf{x}=\mathbf{0}$ if and only if $\mathbf{x}=\mathbf{0}$.

Proof. For a finite abelian group $G$, we can write $G \simeq \mathbb{Z} / k_{1} \mathbb{Z} \oplus \cdots \oplus \mathbb{Z} / k_{M} \mathbb{Z}$ with $k_{i} \mid k_{i-1}(2 \leq$ $i \leq M)$. For $\mathbf{x} \in G^{R}$, we have $\mathbf{x}=\mathbf{x}_{1}+\cdots+\mathbf{x}_{M}$ with $\mathbf{x}_{i} \in\left(\mathbb{Z} / k_{i} \mathbb{Z}\right)^{R}(1 \leq i \leq M)$. Then $Z \mathbf{x}=\mathbf{0}$ is equivalent to $Z \mathbf{x}_{i}=\mathbf{0}(1 \leq i \leq M)$. Fix $i \in \mathbb{N}$ with $1 \leq i \leq M$. Since $\operatorname{gcd}(\operatorname{det} Z, a(G))=1$ and $k_{i} \mid a(G), Z$ is invertible over the ring $\mathbb{Z} / k_{i} \mathbb{Z}$. Hence $Z \mathbf{x}_{i}=\mathbf{0}$ if and only if $\mathbf{x}_{i}=\mathbf{0}$. Thus, $Z \mathbf{x}=\mathbf{0}$ is equivalent to $\mathbf{x}=\mathbf{0}$.

Lemma 3. For $Y=\left(a_{i, j}\right) \in \mathbb{Z}^{R \times S}$ and $L \in \mathbb{N}$ with $L \geq R$, suppose that $G$ is a finite abelian group that is L-coprime to $Y$. Suppose that $A \subseteq G$ for which the equations in (1) are never satisfied simultaneously by distinct elements $x_{1}, \ldots, x_{S} \in A$. Then we have

$$
T(A) \leq C_{1}|A|^{S-R-1},
$$


where $C_{1}=C_{1}(Y)=\left(\begin{array}{c}S \\ 2\end{array}\right)$.

Proof. We have

$$
T(A)=\operatorname{card}\left\{\mathbf{x} \in A^{S} \mid Y \mathbf{x}=\mathbf{0}\right\}
$$

where card $\{V\}$ denotes the cardinality of a set $V$. Since $A \subseteq G$ for which the equations in (1) are never satisfied simultaneously by distinct elements $x_{1}, \ldots, x_{S} \in A$, whenever $Y \mathbf{x}=\mathbf{0}$ for some $\mathbf{x}=\left(x_{1}, \ldots, x_{S}\right) \in A^{S}$, there exist distinct elements $i, j \in\{1, \ldots, S\}$ with $x_{i}=x_{j}$. Fix one of the $C_{1}=\left(\begin{array}{c}S \\ 2\end{array}\right)$ choices of $\{i, j\}$. We consider two cases.

- Case 1: Suppose that $\{i, j\} \cap \mathcal{I}_{Y}(G ; L)=\emptyset$. Since $G$ is $L$-coprime to $Y$, by Lemma 2, we have

$$
\text { card }\left\{\mathbf{x} \in A^{S} \mid x_{i}=x_{j} \text { and } Y \mathbf{x}=\mathbf{0}\right\} \leq|A|^{S-R-1} .
$$

- Case 2: Suppose that $\{i, j\} \cap \mathcal{I}_{Y}(G ; L) \neq \emptyset$. Without loss of generality, we may assume that $j \in \mathcal{I}_{Y}(G ; L)$. Since $G$ is $L$-coprime to $Y$, we can find two disjoint $R$-element subsets $U$ and $V$ of $\{1, \ldots, S\} \backslash\{j\}$ such that the columns of $Y$ indexed by either set form a matrix of determinant coprime to $a(G)$. Since $(U \cup V) \cap\{i, j\} \subseteq\{i\}$ and $U \cap V=\emptyset$, without loss of generality, we may assume that $U \cap\{i, j\}=\emptyset$. It now follows from Lemma 2 that

$$
\text { card }\left\{\mathbf{x} \in A^{S} \mid x_{i}=x_{j} \text { and } Y \mathbf{x}=\mathbf{0}\right\} \leq|A|^{S-R-1} .
$$

On recalling the definition of $C_{1}$ and combining Cases 1 and 2, the lemma follows.

Lemma 4. Let $Y \in \mathbb{Z}^{R \times S}$ satisfy $a_{i, 1}+\cdots+a_{i, S}=0(1 \leq i \leq R)$. For $L, N \in \mathbb{N}$ with $L \geq R$, let $G$ be a finite abelian group with $c(G) \geq N$ that is $L$-coprime to $Y$. Suppose that $A \subseteq G$ for which the equations in (1) are never satisfied simultaneously by distinct elements $x_{1}, \ldots, x_{S} \in A$. Then we have

$$
\sup _{\chi \neq 1}\left|\sum_{x \in A} \chi(x)\right| \leq d(N-1)|G|-|A| .
$$

Proof. This proof can be carried out in the same way as the proof of [7, Lemma 3]. To do this, in the proof of [7, Lemma 3], we set $r_{i}=-1$, and we replace the condition that $G$ is coprime to $\mathbf{r}$ with the condition that $G$ is $L$-coprime to $Y$. We also change the notion of non-trivial solutions in [7] to solutions with distinct coordinates. Finally, we replace the linear equation $r_{1} x_{1}+\cdots+r_{s} x_{s}=0$ with the system of equations (1).

Lemma 5. For $Y=\left(a_{i, j}\right) \in \mathbb{Z}^{R \times S}$ and $L \in \mathbb{N}$ with $L \geq R$, suppose that $G$ is a finite abelian group that is L-coprime to $Y$. Let

$$
Q=Q_{Y}(G ; L)=\left\{B \subseteq \mathcal{I}_{Y}(G ; L)|| B \mid=L-R+1\right\} .
$$

For $B \in Q$, let

$$
\Gamma_{B}=\Gamma_{B, Y}(G ; L)=\left\{\chi=\left(\chi_{1}, \ldots, \chi_{R}\right) \in \hat{G}^{R} \mid \chi_{1}^{a_{1, j}} \cdots \chi_{R}^{a_{R, j}} \neq 1(j \in B)\right\} .
$$

Then we have

$$
\Gamma(G) \subseteq \bigcup_{B \in Q} \Gamma_{B}
$$


Proof. Let $\chi=\left(\chi_{1}, \ldots, \chi_{R}\right) \in \Gamma(G)$. Select any $R$ columns indexed by $\left\{l_{1}, \ldots, l_{R}\right\} \subseteq \mathcal{I}_{Y}(G ; L)$, and we denote by $Z=\left(a_{i, l_{j}}\right)_{1 \leq i, j \leq R}$ the matrix formed by these columns. Suppose that $\chi_{1}^{a_{1, l_{i}}} \cdots \chi_{R}^{a_{R, l_{i}}}=1$ for every $i \in\{1, \ldots, R\}$. Let $\rho$ be an isomorphism from $\hat{G}$ to $G$. It follows that for $1 \leq i \leq R$,

$$
0=\rho(1)=\rho\left(\chi_{1}^{a_{1, l_{i}}} \cdots \chi_{R}^{a_{R, l_{i}}}\right)=a_{1, l_{i}} \rho\left(\chi_{1}\right)+\cdots+a_{R, l_{i}} \rho\left(\chi_{R}\right) .
$$

Write $\rho(\boldsymbol{\chi})=\left(\rho\left(\chi_{1}\right), \ldots, \rho\left(\chi_{R}\right)\right)$. Then the above equation is equivalent to having $\rho(\boldsymbol{\chi}) Z=\mathbf{0}$. Since $G$ is $L$-coprime to $Y$, we have $\operatorname{gcd}(\operatorname{det} Z, a(G))=1$. By Lemma 2 , we have $\rho(\chi)=\mathbf{0}$. It follows that $\chi=\mathbf{1}$, contradicting the fact that $\chi \in \Gamma(G)$.

Since we can find an element $k$ such that $\chi_{1}^{a_{1, k}} \cdots \chi_{R}^{a_{R, k}} \neq 1$ amongst any $R$-element subset of $\mathcal{I}_{Y}(G ; L)$, it follows that there are at least $L-R+1$ values $k \in \mathcal{I}_{Y}(G ; L)$ with $\chi_{1}^{a_{1, k}} \cdots \chi_{R}^{a_{R, k}} \neq 1$. That is, there exists $B \subseteq \mathcal{I}_{Y}(G ; L)$ with $|B|=L-R+1$ such that $\chi \in \Gamma_{B}$. This completes the proof of the lemma.

Lemma 6. Let $Y \in \mathbb{Z}^{R \times S}$ satisfy $a_{i, 1}+\cdots+a_{i, S}=0(1 \leq i \leq R)$. For $L, N \in \mathbb{N}$ with $L \geq R$, let $G$ be a finite abelian group with $c(G) \geq N$ that is $L$-coprime to $Y$. Suppose that $A \subseteq G$ for which the equations in (1) are never satisfied simultaneously by distinct elements $x_{1}, \ldots, x_{S} \in A$. Then we have

$$
|G|^{-R} \sum_{\chi \in \Gamma(G)}\left|F_{1} \cdots F_{S}(\chi)\right| \leq C_{2}(d(N-1)|G|-|A|)^{L-R+1}|A|^{S-L-1},
$$

where $C_{2}=C_{2}(Y ; L)=\left(\begin{array}{c}L \\ L-R+1\end{array}\right)$.

Proof. Let $Q$ and $\Gamma_{B}(B \in Q)$ be defined as in Lemma 5. We have

$$
|G|^{-R} \sum_{\chi \in \Gamma_{B}}\left|F_{1} \cdots F_{S}(\chi)\right| \leq\left(\sup _{\chi \in \Gamma_{B}} \prod_{j \in B}\left|F_{j}(\chi)\right|\right) \cdot|G|^{-R} \sum_{\chi \in \hat{G}^{R}} \prod_{j \notin B}\left|F_{j}(\chi)\right| .
$$

By Lemma 4, we see that for $j \in B$,

$$
\sup _{\chi \in \Gamma_{B}}\left|F_{j}(\chi)\right| \leq d(N-1)|G|-|A| .
$$

Since $G$ is $L$-coprime to $Y$, there are two disjoint $R$-element subsets $U$ and $V$ of $\{1, \ldots, S\} \backslash B$ such that the columns of $Y$ indexed by either set form a matrix of determinant coprime to $a(G)$. Let $Z$ be an $R \times R$ matrix formed by the columns indexed by $U$ (or $V$ ). Note that since $\operatorname{gcd}(\operatorname{det} Z, a(G))=1$, by Lemma 2 , for $\mathbf{y}_{1}, \mathbf{y}_{2} \in A^{R}$, we have $Z \mathbf{y}_{1}=Z \mathbf{y}_{2}$ if and only if $\mathbf{y}_{1}=\mathbf{y}_{2}$. Then by (2), we have

$$
|G|^{-R} \sum_{\boldsymbol{\chi} \in \hat{G}^{R}}\left|\prod_{\substack{j \in U \\(\text { or } j \in V)}} F_{j}(\chi)\right|^{2}=\operatorname{card}\left\{\left(\mathbf{y}_{1}, \mathbf{y}_{2}\right) \in A^{R} \times A^{R} \mid Z \mathbf{y}_{1}=Z \mathbf{y}_{2}\right\}=|A|^{R} .
$$


On combining the above equality with the Cauchy-Schwarz inequality, we see that

$$
\begin{aligned}
& |G|^{-R} \sum_{\chi \in \hat{G}^{R}} \prod_{j \notin B}\left|F_{j}(\chi)\right| \\
\leq & |A|^{S-|B|-2 R} \cdot|G|^{-R} \sum_{\chi \in \hat{G}^{R}}\left|\prod_{j \in U} F_{j}(\chi)\right|\left|\prod_{j \in V} F_{j}(\chi)\right| \\
\leq & |A|^{S-|B|-2 R}\left(|G|^{-R} \sum_{\chi \in \hat{G}^{R}}\left|\prod_{j \in U} F_{j}(\chi)\right|^{2}\right)^{\frac{1}{2}}\left(|G|^{-R} \sum_{\chi \in \hat{G}^{R}}\left|\prod_{j \in V} F_{j}(\chi)\right|^{2}\right)^{\frac{1}{2}} \\
= & |A|^{S-|B|-R} .
\end{aligned}
$$

On combining the above three inequalities, we have

$$
|G|^{-R} \sum_{\chi \in \Gamma_{B}}\left|F_{1} \cdots F_{S}(\chi)\right| \leq(d(N-1)|G|-|A|)^{L-R+1}|A|^{S-L-1}
$$

By Lemma $5, \Gamma(G) \subseteq \bigcup_{B \in Q} \Gamma_{B}$. Since $\left|\mathcal{I}_{Y}(G ; L)\right|=L$, we have $|Q|=\left(\begin{array}{c}L \\ L-R+1\end{array}\right)=C_{2}$. It follows that

$$
|G|^{-R} \sum_{\chi \in \Gamma(G)}\left|F_{1} \cdots F_{S}(\chi)\right| \leq C_{2}(d(N-1)|G|-|A|)^{L-R+1}|A|^{S-L-1} .
$$

This completes the proof of the lemma.

We are now ready to prove Theorem 1.

Proof. (of Theorem 1) This statement will follow by induction. Since $d(N) \leq 1$ and $C>1$, we trivially have that $d(N) \leq\left(\frac{C}{N}\right)^{\frac{L-R+1}{R}}$ whenever $N \leq C$. Let $N>C$, and assume that $d(N-1) \leq\left(\frac{C}{N-1}\right)^{\frac{L-R+1}{R}}$. Let $G$ be a finite abelian group with $c(G) \geq N$ that is $L$-coprime to $Y$. Suppose that $A \subseteq G$ for which $|A|=D(G)$ and the equations in (1) are never satisfied simultaneously by distinct elements $x_{1}, \ldots, x_{S} \in A$. By (3), we have

$$
|G|^{-R}\left|F_{1}(\mathbf{1}) \cdots F_{S}(\mathbf{1})\right|-|G|^{-R} \sum_{\chi \in \Gamma(G)}\left|F_{1} \cdots F_{S}(\chi)\right| \leq T(A) .
$$

On applying Lemmas 3 and 6 , there exist computable constants $C_{1}, C_{2}>0$ such that

$$
|G|^{-R}|A|^{S}-C_{2}(d(N-1)|G|-|A|)^{L-R+1}|A|^{S-L-1} \leq C_{1}|A|^{S-R-1} .
$$

Let $d^{*}(G)=|A||G|^{-1}$. We have

$$
d^{*}(G)^{S}-C_{1} d^{*}(G)^{S-R-1}|G|^{-1}-C_{2}\left(d(N-1)-d^{*}(G)\right)^{L-R+1} d^{*}(G)^{S-L-1} \leq 0 .
$$

We consider two cases.

- Case 1: Suppose that $d^{*}(G)^{S}-C_{1} d^{*}(G)^{S-R-1}|G|^{-1} \leq \frac{1}{2} d^{*}(G)^{S}$. Since $c(G) \geq N$, we have $|G| \geq 2^{N}$, and hence

$$
d^{*}(G) \leq\left(2 C_{1}\right)^{\frac{1}{R+1}}|G|^{-\frac{1}{R+1}} \leq\left(2 C_{1}\right)^{\frac{1}{R+1}} 2^{-\frac{N}{R+1}} .
$$


For $x>0$, the function $2^{-\frac{x}{R+1}} x^{\frac{L-R+1}{R}}$ obtains its maximum of $\left(\frac{(R+1)(L-R+1)}{R e \log 2}\right)^{\frac{L-R+1}{R}}$ when $x=\frac{(R+1)(L-R+1)}{R \log 2}$. Thus, provided that $C \geq \frac{(R+1)(L-R+1)}{R e \log 2}\left(2 C_{1}\right)^{\frac{R}{(R+1)(L-R+1)}}$, we have

$$
d^{*}(G) \leq(C / N)^{\frac{L-R+1}{R}} .
$$

- Case 2: Suppose that $d^{*}(G)^{S}-C_{1} d^{*}(G)^{S-R-1}|G|^{-1}>\frac{1}{2} d^{*}(G)^{S}$. We can deduce from (4) that

$$
d^{*}(G)^{L+1}<2 C_{2}\left(d(N-1)-d^{*}(G)\right)^{L-R+1} .
$$

By setting $C_{3}=\left(2 C_{2}\right)^{-\frac{1}{L-R+1}}$, we have

$$
C_{3} d^{*}(G)^{\frac{L+1}{L-R+1}}+d^{*}(G)<d(N-1) .
$$

Assume that $C \geq \frac{C_{4}}{C_{4}-1}$, where $C_{4}=\left(C_{3}+1\right)^{\frac{R}{L-R+1}}$. Since the function $x^{\frac{L+1}{R}}(x-$ $1)^{-\frac{L-R+1}{R}}-x$ is decreasing for $x>1$, when $N>C$, we have

$$
N^{\frac{L+1}{R}}(N-1)^{-\frac{L-R+1}{R}}-N \leq C^{\frac{L+1}{R}}(C-1)^{-\frac{L-R+1}{R}}-C \leq C C_{3} .
$$

On combining the above two inequalities with the induction hypothesis, we see that

$$
\begin{aligned}
C_{3} d^{*}(G)^{\frac{L+1}{L-R+1}}+d^{*}(G) & <(C /(N-1))^{\frac{L-R+1}{R}} \\
& \leq C_{3}(C / N)^{\frac{L+1}{R}}+(C / N)^{\frac{L-R+1}{R}} .
\end{aligned}
$$

Since the function $C_{3} x^{\frac{L+1}{L-R+1}}+x$ is increasing for $x>0$, we have

$$
d^{*}(G) \leq(C / N)^{\frac{L-R+1}{R}}
$$

On combining Cases 1 and 2, whenever $C \geq \max \left\{\frac{(R+1)(L-R+1)}{R e \log 2}\left(2 C_{1}\right)^{\frac{R}{(R+1)(L-R+1)}}, \frac{C_{4}}{C_{4}-1}\right\}$, we obtain

$$
d(N)=\sup \left\{d^{*}(G) \mid c(G) \geq N \text { and } G \text { is } L \text {-coprime to } Y\right\} \leq(C / N)^{\frac{L-R+1}{R}} .
$$

This completes the proof of Theorem 1 .

Remark 1. Let $Y=\left(a_{i, j}\right) \in \mathbb{Z}^{R \times S}$ satisfy $a_{i, 1}+\cdots+a_{i, S}=0(1 \leq i \leq R)$. For $L, N \in \mathbb{N}$ with $L \geq R$, let $G$ be a finite abelian group with $c(N) \geq N$ that is $L$-coprime to $Y$. Following the notation in [7], we say that a solution $\mathbf{x}=\left(x_{1}, \ldots, x_{S}\right) \in G^{S}$ of (1) is trivial if $x_{j_{1}}=\cdots=x_{j_{l}}$ for some subset $\left\{j_{1}, \ldots, j_{l}\right\} \subseteq\{1, \ldots, S\}$ with $l \geq 2$ and $a_{i, j_{1}}+\cdots+a_{i, j_{l}}=0(1 \leq i \leq R)$. Otherwise, we say a solution $\mathbf{x}$ of $(1)$ is non-trivial. Let $\tilde{D}(G)=\tilde{D}_{Y}(G)$ denote the maximal cardinality of a set $A \subseteq G$ for which (1) has no non-trivial solution with $x_{j} \in A(1 \leq j \leq S)$. Since a solution $\mathbf{x}$ of (1) with distinct coordinates is also a non-trivial solution, we have $\tilde{D}(G) \leq D(G)$. Thus, by Theorem 1 , there exists a positive constant $C=C(Y ; L)$ such that $\tilde{D}(G) \leq|G|(C / N)^{\frac{L-R+1}{R}}$.

Remark 2. Let $Y=\left(a_{i, j}\right) \in \mathbb{Z}^{R \times S}$ satisfy $a_{i, 1}+\cdots+a_{i, S}=0(1 \leq i \leq R)$, and let $G$ be a finite abelian group that is $R$-coprime to $Y$. For $k \in \mathbb{N}$ and $G=\mathbb{Z} / k \mathbb{Z}$, Roth [10] proved that $D(\mathbb{Z} / k \mathbb{Z})=O\left(k /(\log \log k)^{1 / R^{2}}\right)$. By combining his result with Theorem 1 , the proof of $[8$, Corollary 1.3] yields that for a finite abelian group $G$, we have $D(G)=O\left(|G| /(\log \log |G|)^{1 / R^{2}}\right)$. By adapting Bourgain's method in [2], one can significantly improve Roth's bound for $D(\mathbb{Z} / k \mathbb{Z})$ 
by replacing the power of $\log \log k$ with a power of $\log k$. This would lead to a better bound for $D(G)$.

\section{REFERENCES}

[1] T. C. Brown \& J. P. Buhler, A density version of a geometric Ramsey theorem, J. Combin. Theory Ser. A 32 (1982), 20-34.

[2] J. Bourgain, Roth's theorem on progressions revisited, J. Anal. Math. 104 (2008), 155-192.

[3] P. Frankl, R. L. Graham, \& V. Rödl, On subsets of abelian groups with no 3-term arithmetic progression, J. Combin. Theory Ser. A 45 (1987), 157-161.

[4] W. T. Gowers, A new proof of Szemerédi's theorem, Geom. Funct. Anal. 11 (2001), 465-588.

[5] W. T. Gowers, A new proof of Szemerédi's theorem for arithmetic progressions of length four, Geom. Funct. Anal. 8 (1998), 529-551.

[6] D. R. Heath-Brown, Integer sets containing no arithmetic progressions, J. London Math. Soc. 35 (1987), 385-394.

[7] Y.-R. Liu and C. V. Spencer, A generalization of Meshulam's theorem on subsets of finite abelian groups with no 3-term arithmetic progression, Des. Code. Cryptogr. 52 (2009), 83-91.

[8] R. Meshulam, On subsets of finite abelian groups with no 3-term arithmetic progressions, J. Combin. Theory Ser. A 71 (1995), 168-172.

[9] K. F. Roth, On certain sets of integers, J. London Math. Soc. 28 (1953), 104-109.

[10] K. F. Roth, On certain sets of integers (II), J. London Math. Soc. 29 (1954), 20-26.

[11] E. Szemerédi, Integer sets containing no arithmetic progressions, Acta Math. Hungar. 56 (1990), 155-158.

Y.-R. Liu, Department of Pure Mathematics, Faculty of Mathematics, University of Waterloo, Waterloo, Ontario, Canada N2L 3G1

E-mail address: yrliu@math.uwaterloo.ca

C. V. Spencer, Department of Mathematics, Kansas State University, 138 Cardwell Hall, ManHATTAN, KS 66506

E-mail address: cvs@math.ksu.edu

X. Zhao, Department of Mathematics, Huazhong Normal University, Wuhan, Hubei, China 430079

E-mail address: x8zhao@gmail.com 